\title{
Le tragique des guerres d'Italie dans les sermons de Savonarole et de ses émules
}

Il tragico delle guerre d'Italia nei sermoni di Savonarola e dei suoi emuli

The Tragic of Italian Wars in Savonarola's Sermons and in Those of His Imitators

\section{Cécile Terreaux-Scotto}

\section{OpenEdition Journals}

Édition électronique

URL : http://journals.openedition.org/cei/2125

DOI : $10.4000 /$ cei.2125

ISSN : 2260-779X

Éditeur

UGA Éditions/Université Grenoble Alpes

Édition imprimée

Date de publication : 1 novembre 2014

Pagination : 83-109

ISBN : 978-2-84310-285-1

ISSN : $1770-9571$

Référence électronique

Cécile Terreaux-Scotto, « Le tragique des guerres d'Italie dans les sermons de Savonarole et de ses émules », Cahiers d'études italiennes [En ligne], 19 | 2014, mis en ligne le 01 mai 2016, consulté le 26 mars 2021. URL : http://journals.openedition.org/cei/2125; DOI : https://doi.org/10.4000/cei.2125 


\title{
LE TRAGIQUE DES GUERRES D'ITALIE DANS LES SERMONS DE SAVONAROLE ET DE SES ÉMULES
}

\author{
Cécile Terreaux-Scotto \\ Université Grenoble Alpes
}

En 1953, Raffaello Ramat publiait chez Olschki un ouvrage intitulé Il Guicciardini e la tragedia d'Italia. La tragedia d'Italia désigne la période des guerres d'Italie qui ont ravagé la péninsule de I494 à I559, période qui est dominée par ce que Ramat appelle la tirannia del fato (la «tyrannie du destin») — la prédominance du destin dans la causalité d'un événement étant précisément ce qui lui confère son caractère tragique.

Si l'on excepte le récit que Luigi Guicciardini fait du sac de Rome, qu'il qualifie de miseranda tragedia ("misérable tragédie»), dans les textes contemporains aux guerres d'Italie le nom "tragédie» n'apparaît pas. De façon emblématique, Francesco Guicciardini et Benedetto Varchi évoquent les calamità d'Italia (les "calamités d'Italie») ${ }^{3}$. De son côté, le prédicateur dominicain Savonarole emploie le terme tribolazioni ("tribulations»). À lui seul, ce mot inscrit les guerres d'Italie dans un discours religieux. De fait, Savonarole considère que ces événements militaires sont une épreuve physique et spirituelle envoyée par Dieu pour inviter les habitants de la péninsule, et en particulier les Florentins, à faire pénitence.

Qu'elles soient nommées "calamités» ou "tribulations», les guerres d'Italie constituent bien une succession d'événements funestes et effrayants. Autrement dit, elles correspondent à la définition même du "tragique», pris non pas dans son acception littéraire renvoyant au genre de la tragédie, mais comme épithète désignant une façon de percevoir un événement ${ }^{4}$.

I. R. Ramat, Il Guicciardini e la tragedia d'Italia, Florence, Olschki, I953.

2. On lira à ce propos l'intervention de Jean-Claude Zancarini dans ce volume.

3. F. Guicciardini, Storia d'Italia, E. Mazzali (éd.), Milan, Garzanti, I988, vol. I, p. I; B. Varchi, Storia fiorentina, Florence, Salani editore, 1963, vol. I, p. 59.

4. M. Escola (éd.), Le tragique, Paris, Flammarion, 2002. 
Par ailleurs, et surtout, ces événements sont imprévisibles et irrationnels, en fonction des alliances qui se font et se défont et des conquêtes et des défaites qui varient d'un jour à l'autre. Voilà pourquoi les contemporains considèrent que ces guerres sont ordonnées avant tout par la fortune qui domine toutes choses, et en particulier les affaires militaires. De façon significative, la périphrase employée par Francesco Guicciardini pour désigner les guerres d'Italie - il parle des tempi fortunos $i^{5}$ - revient à faire de ces événements, par antonomase, l'incarnation même de la fortune.

Mais pour Savonarole, c'est moins la fortune que la providence qui orchestre les guerres. Et dans l'interprétation qu'il fournit aux Florentins, Dieu a déclenché les guerres pour inciter les hommes à se convertir. Les guerres apparaissent donc moins comme une fatalité écrasante que comme une occasion positive de réformer l'Église.

Dans ses sermons, le prédicateur brosse le tableau terrifiant d'une Italie menacée par une catastrophe imminente, "catastrophe» étant entendue dans le sens tragique du terme, c'est-à-dire comme le dernier événement avant le dénouement d'une action ${ }^{6}$. Cette catastrophe est l'ensemble des fléaux que Dieu menace d'envoyer sur terre avant le renouvellement possible de l'Église. Les Florentins peuvent cependant exercer leur libre arbitre pour échapper aux tribulations : s'ils font pénitence, Dieu les épargnera. De sorte que la prophétie conditionnée que Savonarole développe au fil de ses sermons lui fait écarter une vision tragique de l'Histoire. Ce n'est toutefois qu'au moment de la seconde République florentine, soit trente ans après l'exécution du prédicateur, que les savonaroliens s'en remettront à Dieu pour faire face aux armées espagnoles qui assiègent la cité, comme si la foi pouvait leur épargner un destin tragique.

\section{«L'Italia andrà sottosopra »}

Dès sa prédication dans les années I480 à San Gimignano, Savonarole avait annoncé que l'Église allait être "flagellée» et «renouvelée» (flagellata, rinovata) et que cela ne saurait $\operatorname{tarder}^{7}$. À Florence en I492, il avait ainsi proclamé que le glaive de Dieu était sur le point de s'abattre sur la cité pour la punir de ses péchés. Cette prédiction était exprimée par la for-

5. F. Guicciardini, Dialogo del Reggimento, G. M. Anselmi et C. Varotti (éd.), Turin, Bollati Boringhieri, I994, p. I09.

6. M. Escola, ouvr. cité, p. 227.

7. Sur la prédication des années I483-I485, dont il reste peu de traces, voir G. Cattin, Il primo Savonarola. Poesie e prediche autografe dal Codice Borromeo, Florence, Leo S. Olschki, I973, p. I05-I6I. 
mule bien connue : "Ecce gladius Domini super terram cito et velociter." Les Florentins, qui s'attendaient depuis plusieurs années à une renovatio précédée d'un long temps de tribulations ${ }^{8}$, étaient prêts à entendre et à recevoir le discours prophétique et apocalyptique de Savonarole, d'autant qu'ils étaient habitués à entendre des prophéties — au point que Stéphane Toussaint peut qualifier leur ville de "terre prophétique ${ }^{9}$ ".

C'est pourquoi lorsque le roi de France Charles VIII franchit les Alpes le 2 septembre 1494, Savonarole peut présenter cet événement politique et militaire comme la réalisation de ses prophéties. Dans le premier sermon du cycle sur Aggée, il dit à ce propos : «Tu sais qu’il y a plusieurs années, avant que l'on n'entendît la rumeur ou que l'on ne sentît l'odeur de ces guerres que l'on voit maintenant, commencées par les gens d'outre-monts, de grandes tribulations te furent annoncées ${ }^{\mathrm{IO}}$.»

Cette coïncidence entre les tribulations annoncées et les événements qui se produisent assoit son autorité dans la cité ${ }^{\text {II }}$, si bien qu'il est envoyé en ambassade auprès du roi de France ${ }^{\mathrm{I} 2}$. Il s'agissait en effet de plaider la cause de Florence auprès d'un Charles VIII qui demandait le retour de Pierre de Médicis, alors que la population venait précisément de le chasser. Charles VIII, réceptif à l'argumentation de Savonarole qui le disait choisi par Dieu pour accomplir une grande mission, s'était alors engagé à restituer Pise et les forteresses que lui avait données Pierre de Médicis contre une forte somme d'argent. De sorte que lorsque le souverain avait quitté

8. E. Garin, "L'attesa dell'età nuova e la "renovatio" ", dans L'attesa dell'età nuova nella spiritualità della fine del Medioevo (Convegno del Centro di Studi sulla spiritualità medievale, III, I6-19 octobre 1960), Todi, Presso l'Accademia Tubertina, 1962, en particulier p. I2 et 24; C. Vasoli, "Notizie su Giorgio Benigno Salviati», dans ID., Profezia e ragione. Studi sulla cultura del Cinquecento e del Seicento, Naples, Morano Editore, I974, p. 57-58. Sur la prophétie, O. Niccoli, «Profezie in piazza. Note sul profetismo popolare nell'Italia del primo Cinquecento", Quaderni storici, XIV, mai-août I479, fasc. II, p. 500-539; ID., Profeti e popolo nell'Italia del Rinascimento, Rome, Bari, Gius. Laterza \& Figlia, 1987. La conjonction astrologique de Saturne et de Jupiter en 1484 avait donné lieu à l’annonce de calamités dans l'ensemble de la péninsule : voir C. Vasoli, «Profezie e profeti nella vita religiosa politica fiorentina», dans Magia, astrologia e religione nel Rinascimento (Convegno polacco-italiano, Varsovie, 25-27 septembre 1972), Wroclaw, Warsawa, Kradow, Gdansk, Ossolineum, 1974, p. I7-I9; D. Weinstein, Savonarole et Florence. Prophétie et patriotisme à la Renaissance, s. 1., Calmann-Lévy, I973, p. 95-97.

9. "terra profetica» (S. Toussaint, "Profetare alla fine del Quattrocento», dans G. C. Garfagnini (éd.), Studi savonaroliani. Verso il V centenario, Florence, Sismel Edizioni del Galluzzo, 1996, p. I68).

IO. "Tu sai che più anni fa, innanzi che si sentisse romore o odore alcuno di queste guerre che si veggano ora, mosse dagli oltramontani, che ti furono annunziate gran tribulazioni." (G. Savonarola, Prediche sopra AggeoTrattato circa el reggimento e governo della città di Firenze, L. Firpo (éd.), Rome, Angelo Belardettti, 1965, I, p. I2.) La traduction provient de l'édition suivante: Savonarole, Sermons, écrits politiques et pièces du procès, trad. J.-L. Fournel et J.-C. Zancarini, Paris, Seuil, I993.

II. L. Polizzotto, "The Elect Nation". The Savonarolan Movement in Florence I494-I545, Oxford, Clarendon Press, 1994, p. 2.

I2. R. Ridolf, Vita di Girolamo Savonarola, Florence, Sansoni, I98I, p. I26. On peut lire à ce sujet le témoignage de F. Guicciardini, Storie fiorentine, A. Montevecchi (éd.), Milan, BUR, I998, p. 2 I8. 
Florence le 28 novembre, Savonarole pouvait apparaitre à de nombreux Florentins, selon la formulation de Donald Weinstein, "comme un prophète de Dieu envoyé pour sauver leur ville du cataclysme ${ }^{13}$ ».

De son côté, le prédicateur répète régulièrement à son auditoire que la situation actuelle correspond point par point à ce qu'il avait annoncé dans le passé. Le I3 janvier I495, alors qu'il explique pour quelles raisons le fléau est proche, il insiste par exemple longuement sur le fait qu'il avait entre autres prédit la venue de Charles VIII. Alors que les habitants de la péninsule étaient sceptiques, ils sont forcés aujourd'hui de reconnaître que frère Jérôme disait vrai, et que le nouveau Cyrus qu'il avait annoncé est bel et bien sur le sol de la péninsule :

Souviens-toi quand je te dis, il y a maintenant trois ans, que le vent se lèverait comme dans l'histoire d'Élie et que ce vent balayerait les montagnes. Ce vent est venu et c'était la rumeur qui s'est répandue pendant des années en Italie; et on parlait de ce roi de France, et partout cette rumeur se propageait comme le vent et balayait les montagnes, c'est-à-dire les princes d'Italie, et elle les a incités cette année à croire et à ne pas croire que ce roi doit venir. Et voilà qu'il est venu, et tu disais : - il ne viendra pas, il n'a pas de chevaux, c'est l'hiver -, et moi je me riais de toi car je savais comment les choses allaient se passer. Voilà qu'il est venu, et Dieu a fait de l'hiver un été, comme je te le dis alors. Souviens-toi aussi que je te dis que Dieu irait par delà les montagnes et qu'il le prendrait par la bride et qu'il l'amènerait de ce côté, malgré et contrairement à l'opinion de chacun; et voilà qu'il est venu. Souviens-toi aussi que je t'ai dit que les grandes forteresses et les grands remparts ne vaudraient rien; vois si tout s'est vérifiér ${ }^{14}$.

La répétition anaphorique des impératifs «souviens-toi» (ricordati) est destinée à convaincre les Florentins du bien fondé de sa prédication prophétique et à légitimer sa parole. Toujours dans le même sermon, Savonarole établit d'ailleurs ensuite un récapitulatif de tout ce qu'il avait annoncé, établissant par la même occasion un plaidoyer pro domo qui lui donne implicitement le statut de prophète :

I3. D. Weinstein, ouvr. cité, p. 39.

I4. "[...] ricordati quando io ti dissi, ora sono tre anni, che verrà uno vento a similitudine di quella figura di Elia e che questo vento concuteria li monti. Questo vento è venuto e questo è stato la fama che si sparse anno per Italia; e dicevasi di questo re di Francia, e per tutto questa fama volava come il vento e concuteva i monti, cioè e' principi di Italia, e halli tenuti questo anno commossi in credere e non credere che questo re debbe venire. Ed ecco che è venuto, e tu dicevi: - e’ non verrà, e’ non ha cavalli, egli è il verno -, e io mi ridevo di te che sapevo la cosa come aveva a andare. Ecco che egli è venuto, e Iddio ha fatto del verno state, come allora ti dissi. Ricordati che ancora ti dissi che Iddio andria di là da' monti e che lo pigliaria per la briglia e menerebbelo di qua, a dispetto e contro alla opinione di ciascuno; ed ecco che è venuto. Ricordati ancora che io ti dissi che non varrebbono niente le gran fortezze e le gran mura; vedi se s'è tutto verificato." (G. Savonarola, Prediche sopra i Salmi, V. Romano (éd.), Rome, Angelo Belardetti, I969, III, vol. I, p. 55-56. Sauf indication contraire, c'est nous qui traduisons.) 
Crois-moi, Florence! tu devrais vraiment me croire parce que, de tout ce que je t'ai dit, tu n'as jamais vu changer un iota jusqu'à maintenant, et à l'avenir aussi tu ne verras rien manquer. J'ai prédit, il y a plusieurs années, la mort de Laurent de Médicis, la mort du pape Innocent. De même, l'événement qui vient de se produire, ici à Florence, du changement de cet État. De même, j'ai dit que le jour où le roi de France serait à Pise, le renouveau de cet État aurait lieu ici. Je n'ai pas dit ces choses là-haut en public, mais je les ai dites à ceux qui sont ici, à ce prêche, et j'ai des témoins ici à Florence ${ }^{\mathrm{Is}}$.

Par la suite, dans ses sermons et avec la publication en août I495 du Compendio di rivelazioni, il répétera régulièrement que tout ce qu'il avait annoncé en 1490 est en train de se réaliser ${ }^{16}$, de sorte qu' "il fait de l'histoire de ses prophéties le fondement même de la défense de leur véracitér7 ${ }^{1}$. La réalisation des malheurs qu'il a prédits prédispose dès lors son auditoire à s’inscrire dans une lecture prédéterminée, et par conséquent tragique, des événements.

Adaptant le contenu de ses sermons à l'actualité, à ce qui se passe hic et nunc, Savonarole dessine ainsi la scène tragique d'une Italie mise sens dessus dessous, "sottosopra", selon une formulation qu'il emploiera de façon récurrente à partir du printemps $1495^{18}$. La péninsule est en effet bouleversée par la campagne de Charles VIII qui, avec une rapidité fulgurante, parviendra en quelques mois jusqu’à Naples, suscitant stupeur et désespoir.

Ce sont bien ces sentiments que Francesco Vettori évoque lorsqu'en écrivant la biographie de son père Piero au début du Xvi ${ }^{\mathrm{e}}$ siècle il dit : "Combien de fois je l'ai vu pleurer en ce temps-là, et affirmer qu'il voyait la ruine de la cité et que les Français venaient pour détruire l'Italie tout

I5. "Credimi, Firenze! tu doverresti pure credermi perché, di quello che io tho detto, non hai mai veduto fallire uno iota sino a qui, e ancora per l'avvenire non ne vedrai mancare niente. Io predissi, parecchi anni inanzi, la morte di Lorenzo de’ Medici, la morte di Innocenzio Papa. Item, il caso che è stato adesso, qui a Firenze, della mutazione di questo Stato. Item dissi che quello di, che sarebbe il re di Francia a Pisa, che qui saria la renovazione di questo Stato. Io non ho detto queste cose quassù publice, ma le ho dette a quelli che sono qui a questa predica, e ho li testimonii qui a Firenze." (Ibid., III, vol. I, p. 59)

I6. Ibid., V, vol. I, p. 95; VII, vol. I, p. I23; XXIV, vol. 2, p. II9; G. Savonarola, Prediche sopra Amos e Zaccaria, P. Ghiglieri (éd.), Rome, Angelo Belardetti, I97I, XLVI, vol. 3, p. 336; ID., Prediche sopra Ruth e Michea, V. Romano (éd.), Rome, Angelo Belardetti, I962, XVIII, vol. I, p. 9I ; ID., Prediche sopra Ezechiele, R. Ridolfi (éd.), Rome, Angelo Belardetti, I955, XX, vol. I, p. 258.

I7. J.-L. Fournel, "Le temps de la prophétie dans la Florence savonarolienne (automne I494-été I495)", dans A. Redondo (éd.), La prophétie comme arme de guerre des pouvoirs (XVe-XVII siècles), Paris, Presses de la Sorbonne Nouvelle, 2000, p. 193.

I8. Voir par exemple G. Savonarola, Prediche sopra i Salmi, ouvr. cité, IV, vol. I, p. 8I; VI, vol. I, p. I09; VI, vol. I, p. I23; XIII, vol. I, p. 213; XXVII, vol. 2, p. 6I; ID., Prediche sopra Amos..., ouvr. cité, vol. 2, XXIII, p. I60; ID., Prediche sopra Ruth..., ouvr. cité, XII, vol. I, p. 358 et 370; XX, vol. 2, p. I64; ID., Prediche sopra Ezechiele, ouvr. cité, XIX, vol. I, p. 248. 
entière ${ }^{19}$ !» C'est que Piero Vettori a appartenu à la génération de la paix de Lodi, qui avait permis de stabiliser les rapports entre les différents États de la péninsule et d'assurer une relative tranquillité. Or, comme le décrit Francesco Guicciardini, «à cause du passage des Français, comme à cause d'une soudaine tempête qui met tout sens dessus dessous, l'union de l'Italie se rompit et se déchira ainsi que la pensée et le soin que chacun avait pour les choses communes ${ }^{20} »$. Cette irruption des armées françaises sur le sol italien ne pouvait donc que susciter un profond désarroi face à un avenir incertain.

Il est intéressant de souligner que pour désigner les événements de I494 Guicciardini emploie la même formule lapidaire que Savonarole : l'Italie est sens dessus dessous, "sottosopra». C'est que dans le florilège qu'il établit des sermons de frère Jérôme, Guicciardini insiste justement sur les extraits annonçant les calamités dont sera victime la péninsule ${ }^{21}$. Mais cet emprunt sémantique de Guicciardini à Savonarole souligne aussi et surtout que les Florentins ont à faire face à des événements inouïs et inédits, qui bouleversent les points de repère traditionnels. L'auteur anonyme d'une proposition de réforme rédigée à la demande de la seigneurie en décembre I494 exprime bien cette surprise devant la nouveauté. Il explique que le régime tyrannique des Médicis a été renversé, que chacun aspire à la "liberté» et à «l'égalité», et que Dieu parle par la bouche d'un prophète dont les prédictions viennent de se réaliser. De ce fait, il faut "sortir de l'ordinaire» (uscire dello ordinario), pour faire face à cette situation "extraordinaire» (straordinaria) ${ }^{22}$.

Savonarole prend lui aussi la mesure du caractère exceptionnel des guerres dont il saisit pleinement, et immédiatement, la nouveauté. «Dismoi, avez-vous jamais vu une guerre de ce genre, et prendre les villes de cette façon?", interroge-t-il de façon emblématique le 25 janvier $1495^{23}$. Les anacoluthes, qui font se heurter aussi bien une apostrophe à la deu-

19. "Quante volte lo viddi io in questo tempo piagnere, affermando che vedeva la rovina della città, e ch'e" Franciosi venivano per comune distruzione!» (F. Vettori, Vita di Piero Vettori l'antico scritta di Francesco suo figliuolo, dans E. Niccolini (éd.), Scritti storici e politici, Bari, Gius. Laterza \& Figli, 1972, p. 254.)

20. "[...] per questa passata de' franciosi, come per una subita tempesta rivoltatasi sottosopra ogni cosa, si roppe e squarciò la unione di Italia ed el pensiero e cura che ciascuno aveva alle cose comuni [...]. " (F. Guicciardini, Storie fiorentine..., ouvr. cité, p. 197.)

2I. F. Guicciardini, Estratti savonaroliani, dans R. Palmarocchi (éd.), Scritti autobiografici e rari, Bari, Laterza \& Figli, 1936, vol. 5 .

22. G. Guidi (éd.), Discorso I di anonimo sulla riforma dello stato fiorentino l'anno I494, dans Ciò che accadde al tempo della signoria di novembre dicembre in Firenze l'anno 1494, Florence, Arnaud, 1988, p. 202.

23. "Dimmi, avete voi mai più veduto guerra a questo modo, pigliare le città in questa forma?" (G. Savonarola, Prediche sopra i Salmi, ouvr. cité, VII, p. I23.) 
xième personne du singulier («dis-moi») à un verbe interrogatif à la deuxième personne du pluriel («avez-vous»), ou bien un substantif «une guerre de ce genre» à l'infinitif "prendre» reflètent le chaos suscité par la venue de Charles VIII.

Mais comme l'ont souligné Jean-Louis Fournel et Jean-Claude Zancarini, Savonarole donne un sens à ces événements extraordinaires auxquels Florence est confrontée ${ }^{24}$. Plutôt que de se complaire dans la description tragique des guerres, le prédicateur fournit en effet une clé d'interprétation de ce qui est terrifiant et incompréhensible pour son auditoire. D'une certaine façon, ses sermons vont permettre d'ordonner le désordre, de rationaliser l'irrationnel, de maîtriser l'imprévisible. Il va en somme établir des certitudes dans un contexte où tout n'est qu'incertitude puisque, nous informe Guicciardini, la conquête d'une ville ne prenait "pas des mois mais quelques jours et quelques heures ${ }^{25}$ ”.

Cette certitude énoncée par frère Jérôme est de l'ordre de la prophétie conditionnée, qui incite les Florentins à faire pénitence pour échapper aux tribulations à venir.

\section{«Agite poenitentiam»}

Aux temps rapides des guerres d'Italie - Machiavel parle de guerres "courtes et puissantes ${ }^{26}$ — - Savonarole répond par le temps long de la prophétie.

Il explique à ce propos dans le Compendio di rivelazioni que Dieu, qui seul connaît le moment où s'accomplira la réforme, choisit de révéler ce moment à une personne élue, c'est-à-dire à un prophète qui est seul habilité à annoncer le futur. Or, ce prophète, c'est lui-même, le fraticello de Ferrare ${ }^{27}$. Ainsi se présente-t-il dans les sermons comme le détenteur d'une parole inspirée — «Florence, ce n'est pas moi que tes oreilles ont entendu, mais Dieu ${ }^{28} »$. Et puisque sa parole procède de Dieu ${ }^{29}$, elle ne

24. J.-L. Fournel et J.-C. Zancarini, La politique de l'expérience, Alexandrie, Edizioni dell'Orso, 2002, p. 3I.

25. "non in mesi ma in di ed ore» (F. Guicciardini, Storie fiorentine, ouvr. cité, p. 197).

26. "corte e grosse" (N. Machiavelli, Discorsi sopra la prima deca di Tito Livio, G. Inglese (éd.), Milan, Rizzoli, I996, II, 6, 5).

27. G. Savonarola, Compendio di rivelazioni, testo volgare e latino - Dialogus de veritate prophetica, A. Crucitti (éd.), Rome, Angelo Belardetti, 1974, p. 8-9.

28. "[...] Firenze, hai udito con gli orecchi tuoi non me, ma Iddio." (G. Savonarola, Prediche sopra i Salmi, ouvr. cité, III, vol. I, p. 4I.)

29. G. Savonarola, Prediche sopra Amos..., ouvr. cité, I, vol. I, p. 44. 
peut être qu'infaillible: «[...] ce que je vous ai dit ne bougera pas d'un iota $^{30}$.»

Dans le présent, les Florentins doivent déjà faire face à une réalité au caractère funeste : «Quand je considère l'Italie dans son ensemble et ses villes, je ne vois que ténèbres, je ne vois que tempête, je ne vois que tribulations", déplore Savonarole en novembre $1494^{31}$. Le I5 mars 1495 , il insiste encore : "Tu vois aussi dans quelle situation se trouve l'Italie, qui a déjà commencée à être sens dessus dessous ${ }^{32}$." Le responsable de ce sombre tableau est le roi français Charles VIII, qu'il présente comme le véritable bras armé de Dieu dès le $\mathrm{I}^{\text {er }}$ novembre $\mathrm{I} 494$ : «Dieu envoie ces tribulations et $[. .$.$] Dieu est le chef de ces armées et [...] c'est Lui qui les guide { }^{33}$.»

Mais le pire est encore à venir, comme le promet un Dieu vengeur par la bouche du prédicateur :

Les villes d'Italie seront à feu et à sang. Je les mettrai sens dessus dessous, et je jetterai à terre tes munitions et tes forteresses. Toi, tu as pourtant confiance dans tes remparts, Italie; je détruirai tes remparts, j'abattrai tes forteresses, et je te montrerai que tu devais avoir confiance en moi et non pas dans les choses d'ici bas ${ }^{34}$.

Savonarole annonce à ce propos la venue d'autres armées qui viendront dévaster la péninsule comme le fait déjà l'armée française :

Je te dis qu'un seul barbier ne peut pas raser autant de gens. Un autre barbier viendra. Tu vois que, alors que je t'ai dit ces choses il y a maintenant quatre ans, elles ont avancé; tu devrais aussi croire ce que je te dis et tu ne le crois pas ${ }^{35}$.

Le prédicateur n'indique pas de date précise pour la venue de cet «autre barbier», qui s'inscrit, comme toutes ses prophéties, dans un futur indéterminé. Il explique que les Florentins étant incrédules, il entend faire comme les prophètes des Écritures, qui privaient les incrédules d'infor-

30. "[...] uno iota non mancherà di quello che io v'ho detto." (G. Savonarola, Prediche sopra Giobbe, R. Ridolfi (éd.), Rome, Angelo Belardetti, I957, I, vol. I, p. I5. Voir aussi ID., Prediche sopra Amos..., ouvr. cité, XXIII, vol. 2, p. I60.)

3I. "Quando io considero tutta la Italia e le città di quella, io non veggo se non tenebre, io non veggo se non tempesta, io non veggo se non le tribulazioni.» (G. Savonarola, Prediche sopra Aggeo..., ouvr. cité, III, p. 58.)

32. "Tu vedi pure la Italia in che termini ella si truova, che digià è cominciata tutta andare sottosopra." (G. Savonarola, Prediche sopra Giobbe, ouvr. cité, XIII, vol. I, p. 238.)

33. «Dio manda queste tribulazioni e [...] Dio è el capo di questi eserciti e [...] lui li conduce.» (G. Savonarola, Prediche sopra Aggeo..., I, p. Io. Voir aussi ID., Prediche sopra Giobbe, ouvr. cité, I, vol. I, p. 9.)

34. "Le città della Italia anderanno a foco e fiamma. Io le farò andare sottosopra, e butterò per terra le tue munizioni e le tue fortezze. Tu ti confidi pure in mura, Italia; io guasterò le tue mura, io butterò giù le tue rocche, e monstrerotti che tu dovevi confidarti in me, e non in cose terrene." (G. Savonarola, Prediche sopra Ruth..., ouvr. cité, XV, vol. I, p. 470.)

35. "Io ti dico che uno barbiere solo non può radere tanta gente. Verrà un altro barbiere. Vedi che, avendoti dette queste cose da quattro anni in qua, e' sono andate innanzi; doverresti pur credere quello che io ti dico e tu non credi.» (G. Savonarola, Prediche sopra i Salmi, ouvr. cité, VII, p. I23.) 
mations précises ${ }^{36}$. Il dit aussi que Dieu veut inciter les Florentins à une vigilance constante, comme il apparaît dans un passage des commentaires sur les Psaumes qui mérite d'être intégralement cité malgré sa longueur :

Je t'ai dit: Gladius Domini super terram cito et velociter. Crois-moi, le couteau de Dieu viendra et bientôt. Ne te moque pas de ce «cito", et ne dis pas que c'est un «cito» de l'Apocalypse, qui met une centaine d'années à venir. Crois-moi que c'est pour bientôt : croire ne te nuit en rien, et même t'est utile, car cela te fait revenir à la pénitence et te fait marcher sur le chemin de Dieu; ne pas croire peut te nuire et ne t'est pas utile; aussi crois que le temps est pour bientôt : on ne peut pas dire exactement quand, parce que Dieu ne veut pas, afin que ses élus soient toujours dans la crainte, la foi et la charité, et qu'ils demeurent toujours sur le chemin de Dieu. Et c'est pourquoi je n’ai pas indiqué de temps déterminé, afin que tu fasses toujours pénitence et que tu plaises toujours à Dieu; parce que, par exemple, si on disait aux hommes : - Les tribulations auront lieu d'ici dix ans -, chacun dirait : - Je peux encore attendre un moment avant de me convertir -, et ce serait presque une façon de leur permettre de faire le mal, ce qui ne serait pas convenable. Et c'est pourquoi Dieu ne veut pas qu'on prêche le temps déterminé. Mais je te dis bien ceci, que maintenant c'est le temps de la pénitence; ne vous moquez pas de ce "cito", car je vous le dis : si vous ne faites pas ce que j'ai dit, gare à Florence, gare au peuple, gare au petit, gare au grand ${ }^{37}$ !

Puisque l'objectif est d'amener les Florentins à la pénitence, la temporalité précise des tribulations importe peu, d'autant que se préparer au pire en se convertissant "ne nuit en rien». On peut certes voir dans ces propos la volonté tactique de manipuler un auditoire fragilisé par un contexte difficile - Machiavel, dans une lettre qu’il adressera à Francesco Guicciardini le I7 mai I52I, emploiera d'ailleurs l'adjectif versuto («rusé») pour qualifier frère Jérôme. Mais il y a surtout que la perspective temporelle de Savonarole est celle de Dieu et non de l'homme. Autrement dit, ses sermons s'inscrivent dans l'a-temporalité de l'éternité qui en définitive

36. G. Savonarola, Prediche sopra Amos..., ouvr. cité, I, vol. I, p. 44-45.

37. "Io ti ho detto: Gladius Domini super terram cito et velociter. Credimi che il coltello di Dio verrà e presto. E non ti fare beffe di questo cito, e non dire che e'sia uno cito dello Apocalisse, che sta centinara d'anni a venire. Credimi che sia presto: il credere non ti nuoce niente, anzi ti giova, che ti fa tornare a penitenzia e fatti camminare per la via di Dio; a non credere ti può nuocere e non ti giova; però credi che presto è il tempo: a punto non si può dire, perché Iddio non vuole, acciò che li suoi eletti stieno sempre in timore e in fede e in carità, e stieno sempre nella via di Dio. E però non ti ho detto tempo determinato, acciò che tu facci sempre penitenzia e che tu piacci sempre a Dio; perché, verbigrazia, se si dicessi agli uomini: - La tribulazione ha a venire di qui a dieci anni-, ogni uno direbbe: - Io posso indugiarmi ancora un pezzo a convertirmi -, e saria quasi un dargli licenzia di fare male in quel mezzo, il che saria inconveniente. E però Iddio non vuole che si predichi il tempo determinato. Ma bene ti dico questo, che ora è il tempo della penitenzia; non vi fate beffe di questo cito, ché io vi dico: se non farete quello che io ho detto, guai a Firenze, guai al popolo, guai al piccolo, guai al grande!» (G. Savonarola, Prediche sopra $i$ Salmi, ouvr. cité, III, vol. I, p. 60-6r.) 
ramène tout à l'instant présent, comme il apparaît dans le sermon III sur les Psaumes :

[...] Dieu, fort capable de toute chose, est éternel et embrasse le temps tout entier; parce que chaque chose est présente pour lui, et ce qui fut et ce qui est et ce qui sera est toujours présent pour Lui, et il entend et voit toujours toutes les choses dans le présent ${ }^{38}$.

De même, et de manière significative, lorsque Savonarole parle du futur, il parle aussi du présent et du passé de Florence. Car demander aux Florentins de faire pénitence pour préparer leur salut éternel est une façon de dénoncer, en filigrane, ce qui n'est pas conforme aux vertus chrétiennes dans la Florence d'aujourd'hui et d'hier. «Regarde, dis-je, ton passé; prêtes-y attention et tu verras que, si tu ne changes pas de forme, ce sera toujours de mal en pis ${ }^{39}$ ", met en garde frère Jérôme. Aussi une bonne partie des sermons est-elle consacrée à inviter les Florentins à renoncer au "vieil usage» (vecchia usanza), pour lui préférer une "nouvelle habitude» (nuova consuetudine).

Cependant, en même temps que Savonarole ne fixe aucun délai précis pour la survenue des fléaux qui menacent les Florentins, il martèle que «le temps est proche ${ }^{40} »$, que «le temps est bref ${ }^{41}{ }^{\prime}$. Cette double temporalité, qui associe l'incertitude quant au moment précis de la réalisation de la prophétie, à la certitude que les délais en sont proches, crée une tension tragique à l'intérieur des sermons, comme si les Florentins devaient vivre dans un état d'urgence permanent.

Un passage du sermon XIV sur Aggée est emblématique à cet égard. Voici en quels termes Savonarole annonce la nécessité urgente de faire la paix universelle et d'empêcher la mise en place du gouvernement d'un seul : «Et pressez-vous, car les temps l'exigent, que tu agisses rapidement; et faites-le, parce que, là où il faut des faits, les mots ne suffisent pas ${ }^{42}$." Le prophète est donc celui qui prend la mesure de la conjoncture et qui adapte le contenu de son discours à la «diversité des temps» (diversità dé tempi), ce que Machiavel nommera plus tard la "qualité des temps»

38. "[...] Iddio, capacissimo d'ogni cosa, è eterno e abraccia tutto il tempo; perché ogni cosa a Lui è presente, e ciò che fu è è sarà a Lui sempre è presente, e intende e vede presente sempre ogni cosa." (Ibid., III, vol. I, p. 37)

39. "Guarda, dico, e’ tuoi tempi passati; ponvi cura e vedrai che, se tu non muti forma, sempre anderai di male in peggio. " (G. Savonarola, Prediche sopra Aggeo..., ouvr. cité, XV, p. 260.)

40. "'l tempo è presso" (G. Savonarola, Prediche sopra Amos..., ouvr. cité, VII, vol. I, p. 207. Voir aussi ID., Prediche sopra l'Esodo, P. G. Ricci (éd.), Rome, Angelo Belardetti, I955, XVI, vol. 2, p. II6 et I33).

4I. "el tempo è breve» (G. Savonarola, Prediche sopra Ruth..., ouvr. cité, XIV, vol. I, p. 429. Voir aussi ibid., XVII, vol. 2, p. 70).

42. "E sollecitate, ch'el tempo lo ricerca, che tu facci presto; e fatelo, chè, dove bisogna fatti, non bastano le parole.» (G. Savonarola, Prediche sopra Aggeo..., ouvr. cité, XIV, p. 248.) 
(qualità dè tempi). De plus, la parole du prédicateur qui dit l'urgence de la situation est destinée à émouvoir les Florentins, au sens étymologique du terme, c'est-à-dire à les mettre en mouvement, à les faire agir ${ }^{43}$. C'est pourquoi le dialogue qu'il entretient avec ses auditeurs au fil des sermons est de l'ordre de l'affectif, du pathos - ce qui confere une coloration tragique à sa parole.

De fait, Savonarole, qui cherche à susciter la terreur, privilégie ce que Christian Plantin appelle «l'argumentation par la force», qui "consiste à instaurer un choix qui porte sur les termes également désagréables d'une alternative, l'un de ces termes restant malgré tout plus acceptable que l'autre " ${ }^{4}$. On peut résumer le choix offert par Savonarole aux Florentins à l'aide d'une citation tirée du premier sermon sur Aggée qui remonte au ${ }^{\mathrm{I}}{ }^{\mathrm{er}}$ novembre $\mathrm{I} 494:$ " [...] faites une vraie pénitence [...] autrement je vous annonce que [Dieu] vous punira dans votre âme, dans votre corps et dans votre vie ${ }^{45}$.» D'un côté donc, il s'agit pour les Florentins de renoncer à leurs plaisirs divers et variés; de l'autre, ils risquent une atteinte à leur intégrité non seulement corporelle mais aussi spirituelle. Savonarole évoque en effet comme sanction, d'une part les calamités militaires, auxquelles s'ajouteront la famine et la peste ${ }^{46}$, et d'autre part la damnation éternelle, face à laquelle il présente les catastrophes liées aux guerres comme bien relatives : «[...] considère que les guerres d'Italie, la peste, la faim et toutes les autres tribulations de ce monde ne sont rien en comparaison des peines éternelles ${ }^{47}$.» Dans cette alternative se trouve la conception moderne du tragique telle qu'elle est rappelée par Escola, à savoir le tragique «comme affrontement d'une liberté et d'un destin ${ }^{48}$ ": la liberté de se convertir d'une part, et, en l'absence de pénitence, un destin destructeur d'autre part.

Comme tout argument par la force, il y a un envers, à savoir un "argument par la douceur ${ }^{49}$ »: "Placez votre espérance en Dieu, tournez votre esprit vers la lumière supérieure, voilà ce qui rend les hommes forts en

43. Voir à ce sujet C. Terreaux-Scotto, "Mon dire est un faire” : l'art de persuader dans les sermons politiques de Savonarole», Cahiers d'études italiennes (Filigrana), nº 2, 2005, p. 89-II7.

44. C. Plantin, Essais sur l'argumentation. Introduction à l'étude linguistique de la parole argumentative, Paris, Kimé, 1990, p. 206.

45. "[...] fate una vera penitenzia [...] altramente vi annunzio che [Dio] vi punirà nell'anima, nel corpo e nella vita." (G. Savonarola, Prediche sopra Aggeo..., ouvr. cité, I, p. I5.)

46. G. Savonarola, Prediche sopra Amos..., ouvr. cité, XXVI, vol. 2, p. 223; ID., Prediche sopra Ruth..., ouvr. cité, XII, vol. I, p. 373; ID., Prediche sopra l'Esodo, ouvr. cité, XVIII, vol. 2, p. I9I.

47. "[...] considera che la guerra di Italia, la pestilenzia, la fame e ogni altra tribulazione di questo mondo sono nulla in comparazione delle pene eterne." (G. Savonarola, Prediche sopra i Salmi, ouvr. cité, V, vol. I, p. 83.)

48. M. Escola, ouvr. cité, p. 37.

49. C. Plantin, ouvr. cité, p. 207. 
toute circonstance ${ }^{50}$ ", prévient frère Jérôme. En préférant le Christ aux «choses du monde», les Florentins obtiendront des récompenses, évoquées par une formulation récurrente : leur cité sera "plus puissante, plus riche et plus glorieuse ${ }^{5 \mathrm{I}} »$.

$\mathrm{Au}$ discours comminatoire qui présente les tribulations comme une sanction méritée pour les péchés commis, fait ainsi pendant un discours gratifiant qui exalte la pénitence, source de rédemption et de salut. C'est là toute la fonction de la prophétie conditionnée, qui fait la promesse d'une issue heureuse dans un contexte tragique.

Car, qu'il s'agisse de représailles ou de récompenses, Savonarole présente tout ce qu'il annonce comme une conséquence de l'attitude des hommes. Comme il l'explique dans le Compendio di rivelazioni, sa connaissance du futur "s'appelle prophétie conditionnée de menace ou de promesse, parce qu'il faut comprendre que les choses annoncées s'accompliront si on ne change pas l'ordre des causes dont elles procèdent justement ${ }^{52}{ }^{\prime}$. C'est pourquoi Garfagnini peut écrire que pour Savonarole «ce qui avait le plus de signification, c'était le principe d'une lecture générale de l'histoire, et de ses applications politiques, à l'intérieur d'un dessein divin de salut qui préside à la destinée du monde ${ }^{53}$ ».

Florence est certes une cité sans défense. Avant Machiavel qui écrira dans le chapitre XII du Prince que Charles VIII a pu conquérir la péninsule "avec une craie", frère Jérôme analyse en ces termes les faiblesses structurelles de la défense militaire de la cité :

Ne te souviens-tu pas, Florence, que par le passé je t'ai dit que les grandes forteresses trembleraient, ainsi que les grands remparts, parce que ce serait du gâteau que de les prendre, c'est-à-dire qu'on les prendrait facilement, et que Dieu prendrait les chevaux

50. "cose del mondo [...] Ponete la vostra speranza in Dio, voltate lo intelletto vostro al lume superiore, questo fa gli uomini gagliardi in ogni cosa" (G. Savonarola, Prediche sopra i Salmi, ouvr. cité, XXVI, vol. 2, p. I89).

5I. "più potente, più ricca e più gloriosa". Voir par exemple G. Savonarola, Prediche sopra Aggeo..., ouvr. cité, X, p. I66; XI, p. I87; XV, p. 26I ; XIX, p. 344; ID., Prediche sopra i Salmi, ouvr. cité, II, p. 32 ; XIII, p. 2 I3 et XI, p. I79; ID., Prediche sopra Giobbe, ouvr. cité, I, vol. I, p. I4; ID., Compendio di rivelazioni..., ouvr. cité, p. III. Ces récompenses ne sont d'ailleurs pas seulement d'ordre spirituel. Si Florence est la cité élue et si les Florentins doivent accorder plus d'importance au spirituel qu'au temporel (Prediche sopra i Salmi, ouvr. cité, XXX, vol. 2, p. 235), ils peuvent néanmoins aussi attendre des retombées immédiates et matérielles de leur conversion (ibid., II, vol. I, p. 32).

52. "[...] tal cognizione si domanda profezia condizionata di commminazione ovvero di promissione, perché bisogna intendere che tal cose prenunziate verranno se non si muterà l'ordine delle cause dalle quale ordinatamente hanno a procedere.» (G. Savonarola, Compendio di rivelazioni..., ouvr. cité, p. I20.)

53. "Il massimo del significato era il principio di una lettura generale della storia, e delle sue applicazioni politiche, all'interno di un disegno divino di salvezza che presiede alle sorti del mondo." (G. C. Garfagnini, "Alle origini dell'impegno politico savonaroliano: la profezia", dans ID., Una città e il suo profeta: Firenze di fronte al Savonarola, Florence, Sismel Edizioni del Galluzzo, I998, p. I04-I05.) 
par la bride et qu'il les emmènerait à l'intérieur? Crois-moi, crois-moi, car je te dis qu'il ne te servira à rien d'être dans les grandes tours et les grandes forteresses ${ }^{54}$.

D'un point de vue humain et temporel, les Florentins sont donc condamnés à l'impuissance. Mais ils peuvent se battre avec l'arme de leur foi.

Les menaces brandies par Savonarole prennent dès lors la forme de subordonnées hypothétiques exprimant une condition réalisable. Car comme il l'explique le 28 juillet I495, il n'y a pas de fatalité de la catastrophe : «Italie, je te parle encore une fois et je te dis que ton fléau est conditionné et je t’ai donné la condition : et c'est pourquoi, si tu fais pénitence, tu pourras y remédier; mais si tu ne fais pas pénitence, il ne fait pas de doute que tu n'auras aucun remède ${ }^{55}$." Plus que ce qui va arriver, Savonarole annonce donc ce qui doit arriver si la justice de Dieu n'est pas respectée, ce qui est au cœur même, comme le souligne Paolo Prodi, de la «prophétie politique ${ }^{56} »$.

De sorte que la tension tragique des sermons, qui associe indétermination du temps de Dieu et imminence de la sanction, se dissout dans cette possibilité qu’ont les Florentins d'agir. S’ils changent de vie, alors il seront épargnés. De façon emblématique, Savonarole prévient les Florentins dès le 7 décembre I494: "Ô Florence, si tu agis ainsi que nous l'avons dit, toutes tes affaires iront bien ${ }^{57}$."

Au printemps I495, Savonarole remarquera à ce propos que Dieu a déjà épargnés les Florentins, puisque Charles VIII a continué sa route vers Naples sans dévaster la Toscane. De plus, les réformes institutionnelles de I494, ainsi que la loi du I9 mars I495, qui accorde le pardon aux anciens médicéens et la possibilité pour les condamnés politiques de faire appel devant le Grand Conseil ${ }^{58}$, tout cela a favorisé la clémence divine :

[...] quand le roi de France était à Florence, tout le monde criait : - Sus, sus à Florence! : ils gagneront un grand trésor! - Toi tu n’entendais pas ces choses-là, mais

54. "Non ti ricordi tu, Firenze, che negli anni passati dissi che tremerrieno le gran fortezze, e le gran mura, ché le piglierebbono con le meluzze, cioè facilmente, e che Iddio piglierebbe i cavalli per la briglia e menerebbegli drento? Credimi, credimi, che io ti dico che non ti varrà niente essere nelle gran torri e nelle gran rocche." (G. Savonarola, Prediche sopra i Salmi, ouvr. cité, II, p. 3I.)

55. "Italia, io ti parlo un'altra volta e dicoti che il flagello tuo è condizionato e io tho dichiarata la condizione; e però, se tu farai penitenzia, tu potrai rimediare; ma, se tu non farai penitenzia, senza dubio tu non hai rimedio nessuno.» (G. Savonarola, Prediche sopra i Salmi, ouvr. cité, XXVI, vol. 2, p. I78.)

56. P. Prodi, "Profetismo e utopia nella genesi della democrazia occidentale», dans G. C. Garfagnini (éd.), Savonarola. Democrazia tirannide profezia, Florence, Sismel Edizioni del Galluzzo, 1998, p. 200.

57. La traduction est de Fournel et Zancarini dans Savonarole, Sermons..., ouvr. cité, p. 84. "O Firenze, se tu farai in questo modo che abbiamo detto, le tue cose anderanno tutte bene." (G. Savonarola, Prediche sopra Aggeo..., ouvr. cité, VIII, p. I39.)

58. Sur le texte de la loi et le contexte dans lequel elle a été votée, voir G. Cadoni, «Leggi costituzionali della repubblica fiorentina dal I494 al I5I2", Storia e politica, I980-I984, IV, p. 792-807. 
moi j'étais dans un endroit où je les entendais. Quand il rebroussa chemin, tout le monde disait : - À Florence, à Florence - et Dieu tout puissant les poussa ailleurs. D'un autre côté, si tu n'avais pas fait la réforme, Florence aurait été à feu et à sang et il y aurait eu plus de tyrans qu'auparavant. Miraculeusement donc, toutes les choses se sont faites, et la paix et l'appel et les autres choses ${ }^{59}$.

Et si Dieu a pris la décision de libérer Florence, c'est parce qu'elle avait eu recours à la prière, comme il l'explique en octobre I496 lorsqu'il raconte son ambassade auprès de Charles VIII ${ }^{60}$. Par conséquent, puisque Florence a déjà été épargnée ${ }^{61}$, il faut continuer sur cette voie, pour éviter que Dieu, de sauveur qu'il était, ne se fasse bourreau.

Les hommes disposent en effet de la capacité d'être maîtres de leurs propres actions et de choisir entre le bien et le mal: c'est le libre arbitre, qui permet à l'homme de «choisir et utiliser tous les moyens qui lui sont nécessaires pour le conduire à Dieu, qui ne manque jamais de donner à l'homme une aide suffisante, pour peu qu'il veuille la prendre ${ }^{62} »$. Savonarole précise à cet égard que "Dieu n'a n'obligation ni vis-à-vis de l'homme ni vis-àvis de lui-même, et il lui a même donné le libre arbitre : Et posuit eum in manu consilii sui. Et il lui a montré la voie du salut, pour qu'il puisse la prendre s'il la veut ${ }^{63} »$.

Par conséquent, si les Florentins n'échappent pas aux tribulations qui menacent la péninsule, c'est qu'ils auront choisi, en toute liberté et dans le plein exercice de leur volonté, de ne pas s'en remettre à Dieu. En d'autres termes, loin d'être écrasés par leur propre destin, ils ont la possibilité de l'infléchir.

De ce point de vue, la lecture que Savonarole fait de l'Histoire n'a donc rien de tragique. Les guerres d'Italie apparaissent en effet moins comme une fatalité écrasante que comme une occasion positive de se réformer et de réformer l'Église, de changer le "vieil usage» en "nouvelle coutume»: «les guerres et les fléaux viennent [...] pour le bien des élus de Dieu, c'est-à-dire pour les arracher des mains de ceux qui sont mauvais et de

59. "[...] quando era el re di Francia in Firenze, tutti gridavano: - A sacco, a sacco a Firenze: guadagneranno un gran tesoro! - Tu non udivi queste cose, ma io ero in luogo che l'udivo. Quando tornò indrieto, tutti dicevano: - A Firenze, a Firenze -, e Dio onnipotente li sospinse altrove. Dall'altra parte, se non facevi la riforma, andava Firenze a fuoco e fiamme ed eraci più tiranni che prima. Miracolosamente, adunque, ogni cosa s’e fatto, e la pace e l'appello e le altre cose." (G. Savonarola, Prediche sopra i Salmi, ouvr. cité, XXX, p. 229.)

6o. G. Savonarola, Prediche sopra Ruth..., ouvr. cité, XXVI, vol. 2, p. 325-327.

6I. G. Savonarola, Prediche sopra Aggeo..., ouvr. cité, VIII, p. Iзо.

62. "[...] eleggere e pigliare tutti quelli mezzi che li son necessari a condursi a Dio, el quale non manca mai di dare all'uomo sufficiente adiutorio, purchè voglia pigliarlo." (Ibid., II, p. 30)

63. "[...] Dio non è obbligato all'uomo nè ha obbligo alcuno con seco, anzi gli ha dato el libero arbitrio: Et posuit eum in manu consilii sui. E hagli mostro la via della salute, se lui la vuole, che la possa pigliare." (G. Savonarola, Prediche sopra Giobbe, ouvr. cité, IV, vol. I, p. 62-63.) 
ceux par qui ils sont opprimés et aussi pour les purifier et les faire mériter davantage ${ }^{64}$, affirme-t-il. Les guerres ne sont qu'une étape nécessaire à la gloire universelle de l'Église; aussi est-il inutile d'avoir peur : "Florence, tu as grand peur de la guerre; je te dis : n'aie pas peur, laisse faire la guerre autant qu'on veut, car le Seigneur est avec toi, et il te libère de tout ${ }^{65}$."

Frère Jérôme explique à ce sujet que le renouveau de Florence, "ombilic» et "cœur» de l'Italie ${ }^{66}$, favorisera le renouveau de la péninsule tout entière, puis de l'ensemble de la chrétienté, et que ce renouveau se propagera également chez les Infidèles, qui se convertiront alors au christianisme. Pour cette échéance, Savonarole n'indique pas non plus de date précise, mais il présente le baptême des Infidèles comme un aboutissement certain et inéluctable du processus de réforme :

Je t'ai dit que les Turcs seront baptisés et il en sera ainsi; et s'il n'y avait pas eu ton incrédulité et ton ingratitude, je t’aurais dit non seulement l'année, mais le mois, le jour et l'heure; mais je te ne peux pas te le dire à cause de ton infidélité; crois que les Turcs seront baptisés et parmi ceux qui sont ici il y en a qui le verront ${ }^{67}$.

C'est que le projet de Dieu se réalisera, immanquablement, comme il le dit avec force le 22 février 1497 :

Florence, je t'ai dit beaucoup de choses absolues et beaucoup de choses conditionnées : les fléaux sur l'Italie, le renouveau de l'Église, la conversion des Turcs. Toutes ces choses sont absolues, et vont se produire quoi qu'il arrive et elles ne peuvent absolument pas faire défaut ${ }^{68}$.

Seul le moment où surviendra la réforme, qui aura lieu de manière certaine, est imprécis : "les choses qui, pour Dieu, vont vite peuvent sur terre prendre plus longtemps ", peut-on lire dans les déclarations qui sont attribuées à Savonarole dans les minutes de son procès ${ }^{69}$. Et puisque,

64. "Vengono [...] adunque le guerre e li flagelli per el bene degli eletti di Dio, cioè per cavarli dalle mani de" cattivi e di coloro da chi sono oppressati ed etiam per purgarli e per farli più meritare." (G. Savonarola, Prediche sopra Amos..., ouvr. cité, X, vol. I, p. 284.)

65. "Firenze, tu hai gran paura di guerra; io ti dico: non avere paura, lascia far guerra quanto si voglia, chè il Signore è teco, e libereratti da ogni cosa.» (G. Savonarola, Prediche sopra l'Esodo, ouvr. cité, I, p. 32.)

66. "umbilico" (G. Savonarola, Prediche sopra Aggeo..., ouvr. cité, X, p. I66; "core» (G. Savonarola, Prediche sopra i Salmi..., ouvr. cité, XXII, p. 87).

67. "E' Turchi t'ho detto che s'hanno a battezzare e così sarà; e se non fussi stato la tua incredulità e la tua ingratitudine, io t'arei detto non solamente l'anno, ma il mese, e il di e l'ora; ma io non te lo posso dire per la tua infidelità; credi che e' Turchi s'hanno a battezzare e di quelli che sono qui lo vedranno." (G. Savonarola, Prediche sopra i Salmi, ouvr. cité, XXVI, p. I77. Voir aussi ibid., XI, p. I79.)

68. "Firenze, io ti ho detto molte cose assolute e molte condizionate: el flagello della Italia, la renovazione della Chiesa, la conversione delli turchi. Queste sono tutte assolute, e hanno a venire ad ogni modo e non possono mancare per niente.» (G. Savonarola, Prediche sopra Ezechiele, ouvr. cité, XXII, vol. I, p. 288.)

69. Sur le procès de Savonarole, voir J.-L. Fournel, "Profetismo e dubbi della storia», dans G. C. Garfagnini (éd.), Savonarola. Democrazia..., ouvr. cité, p. 213-229. 
conformément à ce qui est dit dans les Actes des Apôtres (I, 7), le temps de la réforme est le temps de Dieu et non pas le temps de l'homme, la conversion des Infidèles marquera la fin des temps historiques. De sorte que le destin, au sens de succession d'événements marquant la vie d'un homme sans que sa volonté y ait une part active, disparait dans les sermons de Savonarole au profit de la prophétie. Dans ce contexte, il ne peut pas y avoir de fatalité au sens tragique du terme, mais de l'attente confiante dans la providence, la providence étant, conformément à ce qu'enseigne Thomas d'Aquin, la sollicitude affectueuse et bienveillante avec laquelle Dieu prédispose et aide chacun à réaliser ce à quoi il est appelé ${ }^{70}$.

C'est d'ailleurs ainsi que frère Jérôme envisage sa propre destinée ${ }^{71}$. Sa certitude thomiste selon laquelle la prophétie est une grâce donnée gratuitement par Dieu à quelqu'un qu'il choisit, un lumen conférant une connaissance surnaturelle ${ }^{72}$, lui fait souligner à plusieurs reprises que le prophète qu'il est persuadé d'être ${ }^{73}$ n'est qu'un simple instrument entre les mains de Dieu. Voilà pourquoi il peut dire : «Le Seigneur se lèvera et me sauvera et me défendra, et il portera les choses au terme qu'il a fixé. Je n'ai peur de rien et même je te dis que nos choses iront toujours de l'avant ${ }^{74}$."

Selon Savonarole, puisque Dieu accomplira de toute façon ce qu'il a prévu, le prédicateur peut être prêt au sacrifice ultime de sa vie. Un passage des commentaires sur les Psaumes est explicite sur ce point :

Et nous voulons faire deux choses, l'une, combattre, et nous ne cesserons jamais jusqu'à la mort; la seconde, nous voulons vaincre, parce que les affaires du Christ remportent toujours la victoire à la fin. Ne doutez de rien car, même si je meurs, ne doutez pas, dis-je, quà la fin nous remporterons de toute façon la victoire; et ce sera comme l'hydre des poètes, à qui, une fois qu'on a coupé une tête, il en pousse sept autres ${ }^{75}$.

70. B. Mondin, Dizionario enciclopedico del pensiero di san Tommaso d'Aquino, Bologne, Edizioni Studio Domenicano, 2000, ad vocem.

7I. Sur la place de la prophétie dans la vie de Savonarole, voir G. C. Garfagnini, «Profezia e autobiografia: il "caso" Girolamo Savonarola", dans ID., "Questa è la terra tua». Savonarola a Firenze, Florence, Edizioni del Galluzzo, 200o, p. 327-345.

72. G. C. Garfagnini, «Il messaggio profetico di Savonarola e la sua recezione. Domenico Benivieni e Gianfrancesco Pico", dans Studi savonaroliani..., ouvr. cité, p. I99; A. Valerio, "Il profeta e la parola: la predicazione di Domenica da Paradiso nella Firenze post-savonaroliana», dans G. C. Garfagnini, Studi savonaroliani..., ouvr. cité, p. 3or. Sur la prophétie chez Thomas et sa réutilisation par Savonarole, G. C. Garfagnini, "Savonarola e la profezia: tra mito e storia», dans ID., "Questa è la terra tua»..., ouvr. cité, p. 47-52.

73. Sur cette intime conviction, G. C. Garfagnini, "Savonarola e la profezia.... », art. cité, p. 44-45.

74. "El Signore si leverà e mi salverà e defenderà, e condurrà le cose al termine suo. Io non ho paura di niente, anzi ti dico che le cose nostre andranno sempre innanzi." (G. Savonarola, Prediche sopra Ruth..., ouvr. cité, XIX, vol. 2, p. I27.)

75. "E vogliamo fare dua cose, l'una combattere, che non cesseremo mai insino alla morte; la seconda, vogliamo vincere, perché le cose di Cristo hanno sempre nel fine vittoria. Non dubitate di niente che, se bene io morisse, non 
On trouve ainsi dans les sermons plusieurs passages où le prédicateur se dit prêt à mourir $^{76}$, voire réclame la mort, au nom de l'avènement inéluctable de la réforme de Dieu ${ }^{77}$. Le 20 août $\mathbf{1 4 9 6}$, il déclare de façon emblématique : "Je ne veux que ce que tu as donné à tes saints : la mort. Un chapeau rouge, un chapeau de sang : voilà ce que je désire ${ }^{78}$." Moins d'un an plus tard, le II mars I497, il tient des propos semblables : "[...] je veux seulement ta croix, Seigneur. Fais-moi persécuter : je te demande cette grâce, que tu ne me laisses pas mourir dans mon lit, mais que je perde du sang comme tu l'as fait pour moi ${ }^{79}$. " Enfin, près de trois mois avant sa mort, il se dit prêt au martyre ${ }^{80}$. Savonarole choisit donc sa destinée au nom de la supériorité de la providence, tout comme les hommes peuvent ne pas avoir à subir le tragique d'un destin capricieux puisque, d'après lui, ils disposent de leur libre arbitre.

Ses dernières paroles au moment de son exécution vont d'ailleurs dans ce sens. D'après certains témoignages, il aurait crânement répondu à l'évêque qui procédait au rituel de la dégradation qu'il ne pouvait que le séparer de l'Église militante et non de l'Église triomphante, parce que le pouvoir de le séparer de l'Église triomphante ne revenait qu'à Dieu ${ }^{81}$ : le prédicateur tenait à rappeler qu'il mourait en prophète et que les événements tragiques qu'il avait annoncés se réaliseraient malgré sa disparition.

Or, bon nombre de Florentins interpréteront les calamités qui s'abattront sur la péninsule, et en particulier le sac de Rome et le siège de Florence, comme la réalisation des prophéties savonaroliennes.

dubitate, dico, che nello ultimo aremo ogni modo vittoria; e sarà questo come l'idra de poeti che, tagliato uno capo, ne nasce sette." (G. Savonarola, Prediche sopra i Salmi, ouvr. cité, XXVI, p. I84.)

76. Voir par exemple G. Savonarola, Prediche sopra Amos..., ouvr. cité, XVIII, vol. 2, p. 37-38.

77. G. Savonarola, Prediche sopra i Salmi, ouvr. cité, XXVI, vol. 2, p. I8I et ibid., XXII, vol. 2, p. 84.

78. "Non voglio, se non quello che tu hai dato alli tuoi santi: la morte. Uno cappello rosso, uno cappello di sangue: questo desidero.» (G. Savonarola, Prediche sopra Ruth..., ouvr. cité, XIX, p. I26.)

79. "[...] solo voglio la tua croce, Signore. Fammi perseguitare: io ti domando questa grazia, che tu non mi lasci morire in sul letto, ma che io ti renda il sangue come tu hai fatto per me." (G. Savonarola, Prediche sopra Ezechiele, ouvr. cité, XXXIX, vol. 2, p. 195-196.)

8o. G. Savonarola, Prediche sopra l'Esodo, ouvr. cité, VI, vol. I, p. I67.

8I. P. Villari et E. Casanova (éd.), Estratto della Cronaca di Simone Filipepi nuovamente scoperto nell'Archivio Vaticano, dans Scelta di prediche e scritti di fra Girolamo Savonarola con nuovi documenti intorno alla sua vita, Florence, G. C. Sansoni, I898, p. 505; I. Nardi, Istorie della città di Firenze, A. Gelli (éd.), Florence, Successori Le Monnier, I888, vol. I, p. I30. 


\section{«Verrà un altro barbiere »}

Dans le tableau d'une péninsule destinée à être totalement ravagée, Florence et Rome sont toujours citées par Savonarole comme cibles privilégiées de la colère de Dieu. Florence, parce que sa position géographique au cœur de la péninsule la désigne pour être la cité élue de Dieu d'où partira la réforme de l'Église; Rome, parce que la dépravation y est telle qu'il faut détruire cette autre Babylone pour espérer y établir une nouvelle Jérusalem. Le prédicateur présente en effet la ville de la papauté comme la source de la corruption de l'Italie tout entière : «Ô Rome, fille de Sion, tu es le principe des péchés; tu es la reine de toutes les iniquités; tu es la reine de l'orgueil, de la luxure et de tous les vices; tu es le principe et la cause des péchés à la fois des autres prêtres et des autres chrétiens ${ }^{82}$." Rome est d'autant plus coupable qu'en tant que siège de la papauté elle devrait montrer l'exemple à toute la chrétienté. C'est pourquoi frère Jérôme annonce qu'elle "pleurer[a] plus qu'aucune autre ville ${ }^{8_{3}}$ " parce qu'elle «sera la ville la plus fouettée d'Italie ${ }^{84} »$.

À partir du mois de juin 1496, Savonarole précise la nature des dangers qui guettent la péninsule. Une autre armée que celle de Charles VIII viendra dévaster l'Italie au nom de Dieu : «[...] tiens pour certain que Dieu reviendra de toute façon; je ne te dis pas le Roi de France ou d'autres, mais Dieu reviendra de toute façon; et cela ne manquera pas d'arriver, et il viendra avec une armée bien plus grande», dit-il le I2 juin ${ }^{85}$. Plus précisément, cette armée frappera Rome : le 4 septembre 1496, il annonce que les «barbiers" qui viendront raser la péninsule se trouveront sur les rives du Tibre ${ }^{86}$.

De plus, après sa mort, de nombreuses prophéties, énoncées par des laïcs ou des religieux, continuent à circuler. Tous disent que les prédictions de Savonarole auront lieu et que le renouveau succédera à une période de

82. "O Roma, figliuola di Sion, tu sé el principio de' peccati; tu se' la regina di ogni iniquità; tu se' regina di superbia, di lussuria e di ogni vizio; tu se' principio e cagione de peccati e de gli altri preti e de gli altri cristiani." (G. Savonarola, Prediche sopra Ruth..., ouvr. cité, X, vol. I, p. 302.)

83. "piangerai più che alcuna altra città" (G. Savonarola, Prediche sopra Amos..., ouvr. cité, XVI, vol. I, p. 408. Voir aussi ibid., XVII, vol. I, p. 4I7 et Prediche sopra Ruth..., ouvr. cité, XXVII, vol. 2, p. 2Io).

84. "sarà più flagellata delle altre città d'Italia" (G. Savonarola, Prediche sopra Ezechiele, ouvr. cité, XX, vol. I, p. 258).

85. "[...] e tieni per certo che Dio a ogni modo tornerà; non ti dico Re di Francia o altri, ma Dio a ogni modo tornerà; e questo non ha a mancare, e verrà con molto maggiore esercito. " (G. Savonarola, Prediche sopra Ruth..., ouvr. cité, XI, vol. I, p. 325.)

86. Ibid., XXIII, vol. 2, p. 232. 
tribulations ${ }^{87}$. C'est pourquoi le sac de Rome le 6 mai 1527 par les armées espagnoles peut être interprété par les savonaroliens comme la réalisation de la parole de celui qui avait prédit que "des étrangers viendront, qui frapperont et tueront ${ }^{88}$ " et que les églises de Rome "seront transformées en écuries ${ }^{89} "$.

Une autre épreuve frappera encore plus directement les Florentins deux ans plus tard. En juin I529, le pape Clément VII signe les accords de Barcelone et se soumet à l'empereur contre la promesse que les Médicis seront rétablis à Florence. Quelques semaines plus tard, au mois d'août, François Ir $^{\text {er }}$ abandonne ses alliés italiens par le traité de Cambrai, en échange de la libération de ses fils retenus en otages à Madrid. Au mois d'octobre, les troupes de Charles Quint encerclent Florence pour un siège qui durera dix mois. Si bien que les Florentins peuvent voir dans cette succession d'événements la réalisation de la prédiction savonarolienne selon laquelle «l'Italie et Rome seront sens dessus dessous ${ }^{90}$ ».

Or, c'est par la foi en Dieu que la cité choisit de résister à ses ennemis, comme si pour échapper à la violence des armées ennemies, à la peste et à la famine, elle entendait se plier à la prophétie conditionnée formulée par Savonarole. Pendant les trois années de la dernière République, la promesse de fléaux précédant la réforme est en effet entretenue par les frères prêcheurs, frère Zaccaria da Lunigiana, frère Bartolomeo da Faenza et frère Benedetto da Foiano en particulier. Bernardo Segni écrit de manière significative à ce propos que

[...] le gonfalonier et ceux qui gouvernaient la ville tempéraient en partie ces douleurs avec l'espoir que donnaient en chaire un frère de Santa Maria Novella, appelé Foiano, et un frère de San Marco, appelé Zaccheria, qui disaient, en interprétant des prophéties, que bientôt la cité serait victorieuse ${ }^{91}$.

87. O. Niccoli, Profeti e popolo nell'Italia del Rinascimento, Rome, Bari, Gius. Laterza \& Figli, 1987, en particulier p. 15-46; C. Vasoli, "L'attesa della nuova èra in ambienti e gruppi fiorentini del Quattrocento", dans L'attesa dell'età nuova..., ouvr. cité, p. 403; ID., "Temi mistici e profetici alla fine del Quattrocento», dans Studi sulla cultura del Rinascimento, Manduria, Lacaita Editore, 1968, p. 215; D. Weinstein, ouvr. cité, p. 326327, 34I-342, 345-357 et 360-368.

88. "Verrà gente estranea, la quale percoterà e ammazzerà [...] " (G. Savonarola, Prediche sopra Ruth..., ouvr. cité, XIV, p. 430).

89. "saranno fatte stalle di cavalli» (ibid., XII, vol. I, p. 383).

90. "la Italia e Roma andrà sottosopra" (G. Savonarola, Prediche sopra i Salmi, ouvr. cité, XIII, p. 2I3).

91. "[...] il gonfaloniere e li governatori dello stato mitigavano in parte quelli dolori con la speranza, che su per i pergami era data da un frate di Santa Maria Novella, detto il Foiano, e da un frate di San Marco, detto fra Zaccheria, che interpretando profezie, dicevano fra breve tempo la città dover restare vittoriosa." (Storia fiorentina di messer Bernardo Segni gentiluomo fiorentino dall'anno MDXXVII al MDLV colla vita di Niccolò Capponi Gonfaloniere della Republica di Firenze, descritta dal medesimo Segni suo nipote, Milan, Dalla Società Tipografica de' classici italiani, I805-I806, p. I78-I79.) 
Les épreuves sont donc envisagées comme l'ultime punition avant la réforme, comme des tribulations sanctionnant les péchés avant que Dieu n'intervienne pour sauver la cité. Ainsi, dans le sermon que frère Zaccaria prononce pour l'Épiphanie de l'année I530, il établit, comme Savonarole avant lui, un lien de causalité entre les péchés et la sanction divine :

[...] Florence a été pleine de péchés [...] Elle a déjà été plusieurs fois frappée par

Dieu avec de nombreuses tribulations : elle a eu la famine et une grande peste; à présent elle a une guerre qui la broye jusqu'à l'os et pourtant elle ne se convertit pas, elle ne fait pas pénitence, elle ne retourne pas à Dieu ${ }^{92}$.

Et comme frère Jérôme, frère Zaccaria ne pense pas la guerre en termes purement militaires, préférant armer Dieu plutôt que les hommes : «[...] dégaine, Seigneur, ton épée aiguisée de tout côté. Ceci est ta justice : brandis-la contre nos ennemis et finis-en avec eux, afin qu'ils ne puissent plus nous nuire ${ }^{93}$.»

On comprend dès lors pourquoi la prière et les processions propitiatoires peuvent apparaître aux Florentins comme une façon efficace de faire face aux soldats ennemis, d'autant que Savonarole avait proclamé la supériorité de la foi sur les stratégies et les combats militaires : "Italie, tu n'as pas d'autre remède sinon de courir vers le Christ; ne te fie ni aux troupes ni aux remparts, car tu t'illusionnerais ${ }^{94}$.» Une loi du 2 novembre 1529 prescrit d'ailleurs que tous les Florentins inaptes au combat doivent s'agenouiller pour prier dès qu'ils entendent la cloche du palais de la Seigneurie retentir avant une bataille ${ }^{95}$.

Certes, par une loi du 6 novembre 1528, les Florentins choisissent de mettre en place une milice formée de citoyens, à la place des mercenaires qui constituaient depuis très longtemps les forces armées de la ville. Mais de façon éclairante, cette décision politique et militaire est interprétée comme un geste de Dieu qui, dans un élan de compassion, a dissipé une ère d'ignorance aveugle pour aider les Florentins à voir juste et à prendre

92. "[...] Firenze è stata piena di peccati [...] È stata percossa già più volte da Dio con molte tribolationi: ha avuto la carestia et la pestilentia grande; ora ha la guerra che la trita insino all'ossa: et nondimanco non si converte, non fa penitentia, non ritorna a Dio." (Cité par S. Genzano, La seconde république florentine (I527I530). Pensée politique et lectures historiques, thèse de doctorat en études italiennes, sous la direction de JeanClaude Zancarini, ENS de Lyon, 2010, p. I52-153.)

93. "[...] manda fuori, Signore, la spada tua acuta da ogni parte. Questa è la tua iustitia: cavala fuora contro a' nimici nostri, et conclude contro di loro, acciocchè non ci possano nuocere." (Cité par S. Genzano, ouvr. cité, p. I78.) Sur la question de la guerre chez Zaccaria da Lunigiana, voir ses commentaires p. I66-I82.

94. "Italia, tu non hai altro remedio se non correre a Cristo; non ti fidare in squadre né in mura, ché ti becchi el cervello." (G. Savonarola, Prediche sopra i Salmi, ouvr. cité, VII, p. I23.)

95. A. Monti, L'assedio di Firenze (I529-I530). Politica, diplomazia e conflitto durante le Guerre d'Italia, thèse de doctorat en histoire (sous la direction de Franco Angiolini), Université de Pise, 20I3, p. 201. 
les bonnes décisions. Au cours d'une des harangues qui sont prononcées une fois par an pour exhorter les soldats à défendre leur cité, Piero Vettori explique à ce sujet que Dieu "vous a miraculeusement ouvert les yeux pour que vous preniez le seul remède contre vos $\operatorname{maux}^{96}{ }^{\prime}$. Mieux encore, l'orateur Pier Filippo Pandolfini voit dans la milice la réalisation de la prophétie conditionnée de Savonarole; Florence a accepté de se fier à Dieu, elle jouit donc de sa liberté et sera protégée par ses propres habitants :

Cette liberté n’est pas œuvre humaine, cela fait plusieurs années qu'elle a été prédite, et on voit qu'elle est née et qu'elle a été donnée miraculeusement à ce peuple; et aujourd'hui, sur ordre et sous l'impulsion de Dieu, elle se fortifie avec les forces de la cité, afin que la majesté de ce pouvoir soit sûre ${ }^{97}$.

Ainsi, dans la bouche des orateurs, Dieu intervient pour sauver Florence, et les Florentins menacés par l'armée espagnole sont ceux qui jouissent des récompenses terrestres et célestes annoncées trente ans auparavant ${ }^{98}$. Filippo Parenti, chargé lui aussi de galvaniser la cité en lui promettant une victoire certaine, reprend de son côté les affirmations de Savonarole qui faisaient de Florence le point de départ de toute la chrétienté renouvelée : «[...] cette république qui en Italie sera la première à restaurer les anciens ordres militaires, sera celle qui donnera ses lois à ses voisins ${ }^{99}$.»

De façon symptomatique, les harangues se déroulent dans l'église principale de chacun des quatre quartiers, comme s'il fallait mettre la défense de Florence sous la protection divine et rendre grâce au Seigneur. Les Florentins se sont d'ailleurs déjà placés sous la protection de Dieu, puisque le 9 novembre 1528 une majorité écrasante de membres du Grand Conseil a accepté la proposition du gonfalonier de justice Niccolò Capponi d'élire le Christ roi de Florence - seuls i 8 membres sur les I Ioo présents y ont été hostiles ${ }^{100}$. Cette élection a ensuite été renouvelée le 28 juin 1529 et la cité s'est engagée à ne jamais se livrer à un autre souverain que le Christ. En

96. "[...] Dio, dal quale vi furono aperti gl'occhi miracolosamente al pigliar quest'unico rimedio de' vostri mali." (Oratione di Piero Vettori, fatta alla militare ordinanza fiorentina l'anno MDXXIX il di s febbraio, dans R. von Albertini, Firenze dalla repubblica al principato. Storia e coscienza politica, Turin, Einaudi, I995, p. 42I.) 97. "Questa libertà non è opera umana, tanti anni sono che la fu predetta, et vedesi nata et data a questo popolo miracolosamente; et oggi, per ordine et impulsione di Dio, si fortifica con forze cittadinesche, acciò la maestà di questo imperio sia sicura." (Oratione di Pier Filippo di Alessandro Pandolfini al Popolo di Firenze nel tempio di San Lorenzo, a di XXVIII di gennaio MDXXVIII, dans Archivio Storico Italiano, "Documenti per servire alla storia della milizia italiana dal XIII secolo al XVI», s. VI, t. XV, I85I, p. 355.)

98. S. Genzano, ouvr. cité, p. II9-I20.

99. Cité par S. Genzano (ouvr. cité, p. 138), à qui est empruntée la traduction de ce passage : «[...] quella repubblica che in Italia sarà la prima a innovar gli antichi ordini militari, sarà quella che darà le leggi a' suoi vicini." (F. Parenti, "Orazione a' soldati della nuova milizia fiorentina», dans P. Dazzi (éd.), Orazioni politiche del secolo XVI, Florence, G. Barbèra, 1866, p. 465.)

Ioo. C. Roth, L'ultima repubblica fiorentina, Florence, Vallecchi Editore, 1929, p. 78. 
faisant du Seigneur leur souverain, les républicains exauçaient les vœux de Savonarole, qui le 28 décembre 1494 avait affirmé que Dieu voulait donner un seul chef et un seul roi à Florence, et que c'était le Christ ${ }^{\text {ror }}$. S'il s'agissait de montrer qu'ils ne se soumettraient à aucune puissance temporelle, ni à celle de l'empereur ni même à celle du pape ${ }^{\mathrm{IO2}}$, les Florentins choisissaient aussi de s'en remettre à la providence divine. Dans une harangue, Pier Filippo Pandolfini remarque à cet égard que la République «n'est pas gouvernée par la raison ou le conseil humain» et que «les Saints permettent que nous conservions notre liberté ${ }^{\mathrm{IO}}$.

Les républicains sont ainsi convaincus qu'il faut envisager la défense de la liberté de Florence comme une attente confiante dans la providence, qui détournera d'eux le fléau de Dieu. C'est là l'alternative que proposait Savonarole pour échapper à la sanction divine. Varchi raconte qu'

il ne manquait pas de ceux [...] qui, émus par les sermons de frère Jérôme, qu'ils appelaient prophéties, se réjouissaient d'autant plus que des ennemis encerclaient de plus près Florence, car ils étaient persuadés que, quand la cité serait réduite à une telle extrémité, qu'elle n'aurait plus aucun remède et qu'aucune force humaine ne pourrait la défendre en aucune façon, alors finalement, et non avant, seraient envoyés du ciel sur les remparts les anges pour la libérer miraculeusement avec leurs épées ${ }^{104}$.

Les savonaroliens de la seconde république florentine envisagent donc la défense militaire comme un acte de foi. Lanalyse de Francesco Guicciardini à ce propos, même si elle est longue, mérite d'être citée :

Avoir la foi n'est rien d'autre que croire, avec fermeté, presque avec certitude, les choses qui ne sont pas raisonnables, ou, quand elles sont raisonnables, les croire avec une conviction plus forte que ne le voudrait la raison. Celui qui a la foi s'obstine donc en ce qu'il croit, poursuit son chemin, intrépide et résolu, au mépris des difficultés et des périls, et supporte toutes les extrémités [...]. Nous en avons de nos jours un très grand exemple dans cette obstination des Florentins qui, sans espoir d'aucun secours, divisés et en proie à mille difficultés, parce qu'ils attendaient, contre toute raison, la guerre entre le pape et l'empereur, ont retenu sous leurs murailles depuis sept mois déjà toutes les armées, quand nul n'aurait pensé qu'ils aient pu les retenir sept jours; et ils ont même tant fait que, s'ils l'emportaient, plus personne ne s'en étonnerait,

IOI. G. Savonarola, Prediche sopra Aggeo..., ouvr. cité, XXIII, p. 430.

I02. A. Monti, ouvr. cité, p. 199-200.

I03. "[...] non è governata per ragione o consiglio umano [...] i Santi acconsentono al conservare la nostra Libertà» (Oratione di Pier Filippo..., ouvr. cité, p. 350).

I04. "Non vi mancavano di quegli [...] che, dalle parole mossi delle prediche di fra Girolamo, le quali chiamavano profezie, quanto più i nemici stringevano Firenze, tanto si rallegravano essi maggiormente, avendo per fermo che, quando la città fosse in termine ridotta, ch'ella più rimedio nessuno non avesse nè forza umana potesse in verun modo difenderla, allora finalmente, e non prima, dovessero essere mandati dal cielo in sulle mura gli angioli a liberarla miracolosamente colle spade." (B. Varchi, ouvr. cité, vol. 2, p. 154. Voir aussi F. Guicciardini, Storia d'Italia..., ouvr. cité, XX, 2, vol. 3, p. 2263-2264.) 
alors que, auparavant, tous les jugeaient perdus : et cette obstination, la cause en est bien, pour une bonne part, la foi de ne pouvoir périr, selon les prédictions de frère Jérôme de Ferrare ${ }^{\text {Ios. }}$.

C'est que face aux guerres d'Italie, événements terrifiants qui bouleversent les points de repère des habitants de la péninsule, le prédicateur dominicain a refusé une lecture tragique de l'Histoire qui soumettrait l'homme aux caprices du destin. Il a enseigné que chaque homme est libre et a le pouvoir d'infléchir sa propre destinée, mais aussi celle de sa ville, de son pays, et même de toute la chrétienté.

Cette conviction partagée par les savonaroliens n'empêche toutefois pas Florence de devoir céder le I2 août I530 devant les armes temporelles, ces mêmes armes que frère Jérôme avait toujours refusé d'employer — on connaît le jugement de Machiavel selon lequel Savonarole est un "prophète désarmé ${ }^{\mathrm{IO}}$ ». Mais en exaltant le libre arbitre, qui permet aux hommes de ne pas subir le cours tragique de l'Histoire et d'avoir la liberté d'accepter ou de refuser le dessein de Dieu, le dominicain avait anticipé les débats qui allaient bientôt déchirer le monde chrétien.

\section{Bibliographie}

Albertini Rudolf von, Firenze dalla repubblica al principato. Storia e coscienza politica, Turin, Einaudi, 1995.

Cadoni Giorgio, "Leggi costituzionali della repubblica fiorentina dal I494 al I5I2", Storia e politica, I-XIII, I980-I984.

Cattin Giulio, Il primo Savonarola. Poesie e prediche autografe dal Codice Borromeo, Florence, Leo S. Olschki, I973.

Escola Marc (éd.), Le tragique, Paris, Flammarion, 2002.

I05. La traduction est empruntée à J.-L. Fournel et J.-C. Zancarini dans F. Guichardin, Avertissements politiques (I5I2-I530), Paris, Cerf, "La nuit surveillée", I988. "Fede non è altro che credere con openione ferma, e quasi certezza le cose che non sono ragionevole, o, se sono ragionevole, crederle con piu resoluzione che non persuadono le ragione. [...] Esemplo a' di nostri ne è grandissimo questa ostinazione dé fiorentini che essendosi contro a ogni ragione del mondo messi a aspettare la guerra del papa e imperadore sanza speranza di alcuno soccorso di altri, disuniti e con mille difficultà, hanno sostenuto in sulle mura già sette mesi gli eserciti, e quali non si sarebbe creduto che avessino sostenuto sette di, e condotto le cose in luogo che, se vincessino, nessuno più se ne maraviglierebbe, dove prima da tutti erano giudicati perduti: e questa ostinazione ha causata in gran parte la fede di non potere perire secondo le predicazione di Fra Ieronimo da Ferrara." (F. Guicciardini, Ricordi, Milan, BUR, I995, CI, p. 87-89)

I06. Sur cette question, voir J.-C. Zancarini, «Far guerra con la pace nel cuore. La guerra nelle prediche di Girolamo Savonarola», dans G. C. Garfagnini (éd.), Savonarola. Democrazia..., ouvr. cité, p. 43-5ı. 
Estratto della Cronaca di Simone Filipepi nuovamente scoperto nell'Archivio Vaticano, dans P. Villari et E. Casanova (éd.), Scelta di prediche e scritti di fra Girolamo Savonarola con nuovi documenti intorno alla sua vita, Florence, G. C. Sansoni, I898, p. 453-5I8.

Fournel Jean-Louis, "Profetismo e dubbi della storia», dans G. C. Garfagnini (éd.), Savonarola. Democrazia tirannide profezia, Florence, Sismel Edizioni del Galluzzo, 1998, p. 213-229.

—, «Le temps de la prophétie dans la Florence savonarolienne (automne I494-été I495)", dans A. Redondo (éd.), La prophétie comme arme de guerre des pouvoirs (XVe-XVII siècles), Paris, Presses de la Sorbonne Nouvelle, 2000.

Fournel Jean-Louis et Zancarini Jean-Claude, La politique de l'expérience, Alexandrie, Edizioni dell'Orso, 2002, p. 3I.

Garin Eugenio, "L'attesa dell'età nuova e la "renovatio" ", dans L'attesa dell'età nuova nella spiritualità della fine del Medioevo (Convegno del Centro di Studi sulla spiritualità medievale, III, I6-19 octobre 1960), Todi, Presso l'Accademia Tubertina, I962, p. 9-35.

Garfagnini Gian Carlo, «Il messaggio profetico di Savonarola e la sua recezione. Domenico Benivieni e Gianfrancesco Pico ", dans ID. (éd.), Studi savonaroliani. Verso il V centenario, Florence, Sismel Edizioni del Galluzzo, 1996, p. 197-2II.

—, "Alle origini dell'impegno politico savonaroliano: la profezia", dans ID., Una città e il suo profeta: Firenze di fronte al Savonarola, Florence, Sismel Edizioni del Galluzzo, 1998, p. 43I-44I.

—, "Profezia e autobiografia: il "caso" Girolamo Savonarola», dans ID., "Questa è la terra tua». Savonarola a Firenze, Florence, Edizioni del Galluzzo, 2000, p. 327-345.

—, "Savonarola e la profezia: tra mito e storia», dans ID., "Questa è la terra tua». Savonarola a Firenze, Florence, Edizioni del Galluzzo, 2000, p. 29-55.

Genzano Silvia, La seconde république florentine (I527-I530). Pensée politique et lectures historiques, thèse de doctorat en études italiennes, sous la direction de Jean-Claude Zancarini, ENS de Lyon, 20I0, 327 p.

Guicciardini Francesco, Estratti savonaroliani, dans R. Palmarocchi (éd.), Scritti autobiografici e rari, Bari, Laterza \& Figli, I936, vol. 5.

—, Storia d'Italia, E. Mazzali (éd.), Milan, Garzanti, I988.

—, Dialogo del Reggimento, G. M. Anselmi et C. Varotti (éd.), Turin, Bollati Boringhieri, I994.

—, Ricordi, Milan, BUR, I995.

—, Storie fiorentine, A. Montevecchi (éd.), Milan, BUR, 1998. 
Guid G Guidubaldo (éd.), Discorso I di anonimo sulla riforma dello stato fiorentino l'anno I494, dans ID., Ciò che accadde al tempo della signoria di novembre dicembre in Firenze l'anno 1494, Florence, Arnaud, 1988.

Machiavelli Niccolò, Discorsi sopra la prima deca di Tito Livio, G. Inglese (éd.), Milan, Rizzoli, 1996.

Mondin Battista, Dizionario enciclopedico del pensiero di san Tommaso d'Aquino, Bologne, Edizioni Studio Domenicano, 2000.

Monti Alessandro, L'assedio di Firenze (I529-I530). Politica, diplomazia e conflitto durante le Guerre d'Italia, thèse de doctorat en histoire (sous la direction de Franco Angiolini), Université de Pise, 2013, 442 p.

Nardi Iacopo, Istorie della città di Firenze, A. Gelli (éd.), Florence, Successori Le Monnier, I888, 2 vol.

Niccoli Ottavia, "Profezie in piazza. Note sul profetismo popolare nell'Italia del primo Cinquecento", Quaderni storici, XIV, mai-août I479, fasc. II, p. 500-539.

—, Profeti e popolo nell'Italia del Rinascimento, Rome, Bari, Gius. Laterza \& Figlia, 1987.

Oratione di Pier Filippo di Alessandro Pandolfini al Popolo di Firenze nel tempio di San Lorenzo, a dì XXVIII di gennaio MDXXVIII, dans Archivio Storico Italiano, "Documenti per servire alla storia della milizia italiana dal XIII secolo al XVI», s. VI, t. XV, I85I, p. 350-376.

Parenti Filippo, "Orazione a' soldati della nuova milizia fiorentina", dans P. Dazzi (éd.), Orazioni politiche del secolo XVI, Florence, G. Barbèra, I866.

Plantin Christian, Essais sur l'argumentation. Introduction à l'étude linguistique de la parole argumentative, Paris, Kimé, I990.

Polizzotto Lorenzo, "The Elect Nation". The Savonarolan Movement in Florence I494-I545, Oxford, Clarendon Press, 1994.

Prodi Paolo, «Profetismo e utopia nella genesi della democrazia occidentale», dans G. C. Garfagnini (éd.), Savonarola. Democrazia tirannide profezia, Florence, Sismel Edizioni del Galluzzo, 1998, p. I99-2II.

RAмAт Raffaello, Il Guicciardini e la tragedia d'Italia, Florence, Olschki, I953.

Ridolfi Roberto, Vita di Girolamo Savonarola, Florence, Sansoni, I98I. Rотн Cecil, L'ultima repubblica fiorentina, Florence, Vallecchi Editore, I929.

Savonarola Girolamo, Prediche sopra Ezechiele, R. Ridolfi (éd.), Rome, Angelo Belardetti, 1955.

—, Prediche sopra l'Esodo, P. G. Ricci (éd.), Rome, Angelo Belardetti, 1955.

—, Prediche sopra Giobbe, R. Ridolfi (éd.), Rome, Angelo Belardetti, I957. 
-, Prediche sopra Ruth e Michea, V. Romano (éd.), Rome, Angelo Belardetti, 1962.

-, Prediche sopra Aggeo - Trattato circa el reggimento e governo della città di Firenze, L. Firpo (éd.), Rome, Angelo Belardettti, 1965.

-, Prediche sopra i Salmi, V. Romano (éd.), Rome, Angelo Belardetti, I969.

—, Prediche sopra Amos e Zaccaria, P. Ghiglieri (éd.), Rome, Angelo Belardetti, I97I.

-, Compendio di rivelazioni, testo volgare e latino - Dialogus de veritate prophetica, A. Crucitti (éd.), Rome, Angelo Belardetti, I974.

-, Sermons, écrits politiques, et pièces du procès, trad. J.-L. Fournel et J.-C. Zancarini, Paris, Seuil, 1993.

Storia fiorentina di messer Bernardo Segni gentiluomo fiorentino dall'anno MDXXVII al MDLV colla vita di Niccolò Capponi Gonfaloniere della Republica di Firenze, descritta dal medesimo Segni suo nipote, Milan, Dalla Società Tipografica de’ classici italiani, I805-I806.

Terreaux-Scotto Cécile, "Mon dire est un faire" : l'art de persuader dans les sermons politiques de Savonarole", Cahiers d'études italiennes (Filigrana), no 2, 2005, p. 89-II7.

Toussaint Stéphane, «Profetare alla fine del Quattrocento», dans G. C. Garfagnini (éd.), Studi savonaroliani. Verso il V centenario, Florence, Sismel Edizioni del Galluzzo, 1996, p. I67-I8I.

VAlerio Adriana, «Il profeta e la parola: la predicazione di Domenica da Paradiso nella Firenze post-savonaroliana», dans G. C. Garfagnini (éd.), Studi savonaroliani. Verso il V centenario, Florence, Sismel Edizioni del Galluzzo, 1996, p. 299-397.

VArChi Benedetto, Storia fiorentina, Florence, Salani editore, 1963.

VAsoli Cesare, «L'attesa della nuova èra in ambienti e gruppi fiorentini del Quattrocento", dans L'attesa dell'età nuova nella spiritualità della fine del Medioevo (Convegno del Centro di Studi sulla spiritualità medievale, III, I6-I9 octobre 1960), Todi, Presso l'Accademia Tubertina, I962, p. $370-432$.

—, "Temi mistici e profetici alla fine del Quattrocento", dans ID., Studi sulla cultura del Rinascimento, Manduria, Lacaita Editore, 1968, p. I80240.

—, "Notizie su Giorgio Benigno Salviati», dans ID., Profezia e ragione. Studi sulla cultura del Cinquecento e del Seicento, Naples, Morano Editore, I974, p. I7-I27.

—, "Profezie e profeti nella vita religiosa politica fiorentina», dans Magia, astrologia e religione nel Rinascimento (Convegno polacco-italiano, Var- 
sovie, 25-27 septembre 1972), Wroclaw, Warsawa, Kradow, Gdansk, Ossolineum, 1974, p. 16-29.

Vettori Francesco, Vita di Piero Vettori l'antico scritta di Francesco suo figliuolo, dans E. Niccolini (éd.), Scritti storici e politici, Bari, Gius. Laterza \& Figli, 1972, p. 249-257.

Weinstein Donald, Savonarole et Florence. Prophétie et patriotisme à la Renaissance, s. l., Calmann-Lévy, 1973.

ZANCARINI Jean-Claude, «Far guerra con la pace nel cuore. La guerra nelle prediche di Girolamo Savonarola», dans G. C. Garfagnini (éd.), Savonarola. Democrazia tirannide profezia, Florence, Sismel Edizioni del Galluzzo, 1998, p. 43-51. 